\title{
A FREE BOUNDARY PROBLEM ARISING FROM SORPTION OF SOLVENTS IN GLASSY POLYMERS*
}

\author{
BY
}

\author{
DANIELE ANDREUCCI AND RICCARDO RICCI ${ }^{1}$ \\ Istituto Matematico "Ulisse Dini"
}

1. Introduction. In this paper we study a model for the process of sorption of solvents by glassy polymers. This model, proposed in 1978 by Astarita and Sarti [1], differs from all previous attempts by the fact that it stresses the presence of a free boundary. The role of the free boundary is to simulate a sharp morphological discontinuity in the material between a penetrated zone, with a relatively high solvent content, and a glassy region, where the solvent concentration is negligibly small and is actually taken to be zero in the model. The solvent is supposed to diffuse in the penetrated zone according to Fick's law. On the free boundary one assumes mass conservation and an empirical law accounting for the following facts: (i) no penetration occurs if the solvent concentration is less than some threshold value, and (ii) above such a threshold the speed of penetration increases with the value of the concentration on the free boundary. A typical law for penetration dynamics, in the one-dimensional case, is given by

$$
\dot{s}(t)=k\left|c(s(t), t)-c^{*}\right|^{m}
$$

(see [1,5]), where $k$ and $m$ are positive constants, $x=s(t)$ represents the free boundary, and $c(x, t)$ and $c^{*}$ are the solvent concentration and the threshold concentration, respectively. In [4] the authors give a complete theoretical analysis of the problem, assuming that the concentration remains constant at the fixed boundary. In particular, they show that the solution of this problem accounts for the qualitative behavior observed in sorption experiments.

Here we investigate the sorption model assuming a flux condition at the fixed boundary, still confining our analysis to the one-dimensional case. The most interesting case, from both the application and the mathematical point of view, occurs when the polymer is initially unpenetrated, i.e., when $s(0)=0$. In this case a fixed point argument of the type used in [4] fails in proving locai existence because of the lack of estimate of higher-order

\footnotetext{
${ }^{*}$ Received January 8, 1985.

${ }^{1}$ Work partially supported by USA-ERO contract DAJA 45-83-C-0053.
} 
derivatives of the solution. However, a local solution can be constructed as a limit of an approximating sequence of problems in which the polymer is initially penetrated, i.e., $s_{n}(0)=b_{n}$ for positive $b_{n}$, and letting $b_{n}$ tend to zero.

Uniqueness of the solution follows from a comparison argument based on time translation. We emphasize that uniqueness is obtained by using the minimal hypothesis of regularity for which the problem makes sense.

2. Existence and uniqueness of the solution. Here we consider the abstract free boundary problem arising from the model sketched in Sec. 1. Using nondimensional variables $x, t$ and indicating by $c$ the (nondimensional, normalized) excess of concentration with respect to the threshold value $q$, we must solve the following:

Problem P. Find $T>0, s \in C^{1}[0, T], c \in C^{2,1}\left(D_{T}\right) \cap C^{1,0}\left(\bar{D}_{T}\right)$, where $D_{T}=\{(x, t): 0$ $<x<s(t), 0<t<T\}$, such that

$$
\begin{gathered}
c_{x x}-c_{t}=0 \quad \text { in } D_{T}, \\
s(0)=0, \\
c_{x}(0, t)=g(t), \quad 0<t<T, \\
\dot{s}(t)=f(c(s(t), t)), \quad 0<t<T, \\
c_{x}(s(t), t)=-(q+c(s(t), t)) \dot{s}(t), \quad 0<t<T,
\end{gathered}
$$

where $q$ is a nonnegative constant.

According to the analysis of Sec. 1, we assume that

$$
f \in C^{0}[0,+\infty) \cap C^{1}(0,+\infty), \quad f(0)=0, f^{\prime}(y)>0, y>0 .
$$

Moreover, we assume that

$$
g \in C^{1}[0,+\infty), \quad g(t) \leqslant 0, t>0, g(0)<0 .
$$

Let us start our analysis by proving the uniqueness of the solution.

Lemma 2.1. Let $(c, s, t)$ be a solution of $P$. Then

$$
\begin{array}{cl}
c(x, t)>0 & \text { in } D_{T} ; \quad \dot{s}(t)>0, \quad 0<t<T ; \\
& c_{x}(x, t)<0 \quad \text { in } D_{T} .
\end{array}
$$

Proof. Since $c_{x}$ is assumed to be continuous in $\bar{D}_{T}$, we have $c(0,0)=c_{0}$, where $c_{0}$ is the unique positive solution of

$$
g(0)=-\left(q+c_{0}\right) f\left(c_{0}\right) .
$$

Suppose that $c(x, t)<0$ somewhere in $D_{T}$ and let $\bar{t}=\inf \{t>0: c(x, t)<0$ for some $x \in[0, s(t)]\}$. Then $c(s(\bar{t}), \bar{t})=0$ and $(s(\bar{t}), \bar{t})$ is a minimum point in $D_{T} \cap\{(x, t), t \leqslant \bar{t}\}$. Moreover, $c_{x}(s(\bar{t}), \bar{t})=0$ contradicting the boundary point principle. Inequality (2.9) follows from the maximum principle.

Theorem 1. Problem $P$ admits at most one solution.

Proof. Let $(c, s, t)$ be a solution of $(P)$. Define a new function $u(x, t)$ by the following transformation:

$$
u(x, t)=-\int_{x}^{s(t)}\{c(\xi, t)+q\} d \xi
$$


It is easy to check that $(u, s, T)$ solves the following free boundary problem:

$$
\begin{gathered}
u_{x x}-u_{t}=0 \quad \text { in } D_{T}, \\
u(0, t)=\int_{0}^{t} g(\tau) d \tau, \quad 0<t<T, \\
u(s(t), t)=0, \quad 0<t<T, \\
s(0)=0, \quad \dot{s}(t)=f\left(u_{x}(s(t), t)-q\right), \quad 0<t<T .
\end{gathered}
$$

Suppose now that $\left(c_{1}, s_{1}, T_{1}\right)$ and $\left(c_{2}, s_{2}, T_{2}\right)$ are two (maximal) solutions of $(P)$, and define $u_{i}, i=1,2$, according to $(2.10)$.

Let $u_{\varepsilon}(x, t)=u_{2}(x, t+\varepsilon), s_{\varepsilon}(t)=s_{2}(t+\varepsilon), \varepsilon>0$. Suppose that $s_{\varepsilon}(t) \leqslant s_{1}(t)$ somewhere, and let $\bar{t}$ be the first time where $s_{\varepsilon}(t)=s_{1}(t)$. Consider $v(x, t)=u_{\varepsilon}(x, t)-u_{1}(x, t)$ in $\left\{(x, t): 0<x<s_{1}(t), 0<t<\bar{t}\right\}$.

Then we have

$$
\begin{gathered}
v(0, t)=\int_{-\varepsilon}^{t} g(r+\varepsilon) d r-\int_{0}^{t} g(r) d r=\int_{t}^{t+\varepsilon} g(r) d r<0, \\
v(x, 0)=u_{\varepsilon}(x, 0)=u_{2}(x, \varepsilon)<0, \\
v\left(s_{1}(t), t\right)=u_{\varepsilon}\left(s_{1}(t), t\right)<0, \quad 0<t<\bar{t} .
\end{gathered}
$$

Then $\left(s_{\varepsilon}(\bar{t}), \bar{t}\right)$ is a maximum point for $v$ and $v_{x}(s(\bar{t}), \bar{t})=f^{-1}\left(\dot{s}_{\varepsilon}(\bar{t})\right)-f^{-1}\left(\dot{s}_{1}(\bar{t})\right) \leqslant 0$, contradicting the boundary point principle.

It follows that $s_{\varepsilon}(t)>s_{1}(t)$ for any $t$. Analogously, if we put $s_{-\varepsilon}(t)=s_{2}(t-\varepsilon)$, we have $s_{-\varepsilon}(t)<s_{1}(t)$. For any $t<T_{2}, \lim _{\varepsilon \rightarrow 0} s_{-\varepsilon}(t)=\lim _{\varepsilon \rightarrow 0} s_{\varepsilon}(t)=s_{2}(t)$, and so $s_{2}(t)=$ $s_{1}(t)$ for any $t$.

To prove the existence of a local solution of problem $(P)$ we construct a sequence of solutions $\left(c_{n}, s_{n}, T_{n}\right)$ for the approximating problems $\left(P_{n}\right)$ obtained by substituting condition (2.2) by

$$
\begin{gathered}
s_{n}(0)=b_{n}>0, \\
c_{n}(x, 0)=h_{n}(x), \quad 0<x<b_{n} .
\end{gathered}
$$

We choose $\left\{b_{n}\right\}$ to be a decreasing sequence, $b_{n} \rightarrow 0$, and

$$
\begin{aligned}
h_{n}(x)=c_{0}-\left(q+c_{0}\right) f & \left(c_{0}\right)\left(x-b_{n}\right) \\
& +\alpha_{n}\left(x-b_{n}\right)^{2}+\beta_{n}\left(x-b_{n}\right)^{3},
\end{aligned}
$$

where $c_{0}$ is the same as in Lemma 2.1, and $\left(\alpha_{n}, \beta_{n}\right)$ is the unique solution of the linear system obtained by imposing two more conditions. The first one is simply $h_{n}^{\prime}(0)=g(0)$. To obtain the second equation we differentiate (2.4) and (2.5) with respect to time and eliminate $\ddot{s}_{n}$; then in the resulting equation we make the substitutions $c_{n t}\left(b_{n}, 0\right)=h_{n}^{\prime \prime}\left(b_{n}\right)$ and $c_{n t x}\left(b_{n}, 0\right)=h_{n}^{\prime \prime \prime}\left(b_{n}\right)$.

$$
\frac{2}{3} c_{0} \leqslant h_{n}(x) \leqslant 2 c_{0}, \quad \forall n, 0 \leqslant x \leqslant b_{n} .
$$

We now establish some a priori estimates for the solutions of problems $\left(P_{n}\right)$. 
The arguments used in Lemma 2.1 give

$$
\begin{gathered}
c_{n}(x, t)>0 \text { in } D_{T_{n}}=\left\{(x, t): 0<x<s_{n}(t), 0<t<T_{n}\right\}, \\
\dot{s}_{n}(t)>0,0 \leqslant t \leqslant T_{n}, \\
c_{n_{x}}(x, t)<0 \text { in } D_{T_{n}} .
\end{gathered}
$$

To get further estimates we need an a priori bound for $s_{n}(t)$. If $q>0$, integrating Green's identity, we have

$$
s_{n}(t)=b_{n}+\frac{1}{q}\left[\int_{0}^{b_{n}} h_{n}(x) d x-\int_{0}^{s_{n}(t)} c_{n}(x, t)-\int_{0}^{t} g(r) d r\right] .
$$

This gives the requested bound

$$
s_{n}(t) \leqslant b_{n}\left(1+\frac{2 c_{0}}{q}\right)+\frac{g_{T}}{q} t, \quad 0 \leqslant t \leqslant T,
$$

where $g_{T}=\|g\|_{C^{0}[0, T]}$. If $q=0$, we have

$$
s_{n}(t) \leqslant b_{n}+f\left(2 c_{0}\right) t=S_{n}(t), \quad 0 \leqslant t \leqslant T^{\prime}
$$

where $T^{\prime}$, independent of $n$, is such that $\|g\|_{C^{\prime \prime}\left[0, T^{\prime \prime}\right]} \leqslant 2 C_{0} f\left(2 c_{0}\right)$ (notice that $T^{\prime}>0$ ). The proof of $\left(2.20^{\prime}\right)$ follows by comparing our solution $\left(c_{n}, s_{n}\right)$ to the explicit solution $\left(C_{n}, S_{n}\right)$, where $C_{n}(x, t)=2 c_{0} \exp \left[-f\left(2 c_{0}\right)\left(x-b_{n}\right)+f^{2}\left(2 c_{0}\right) t\right]$, using the same technique as in the proof of Theorem 1.

As a consequence of $(2.20)$ and $\left(2.20^{\prime}\right)$, we have

Lemma 2. There exist two constants $C(T), G(T)$, independent of $n$, such that

$$
\begin{aligned}
c_{n}(x, t) & \leqslant C(T), \quad c_{n x}(x, t) \geqslant G(T), \\
& \text { in } D_{n}(T)=\left\{(x, t): 0<x<s_{n}(t), 0<t<T\right\}
\end{aligned}
$$

(for $q=0, T \leqslant T^{\prime}$ ).

Proof. The first inequality is obtained, if $q>0$, by comparing $c_{n}(x, t)$ with the heat polynomial

$$
J_{n}(x, t)=\left(g_{T}^{2} / 2 q\right) x^{2}-g_{T} x+\left(g_{T}^{2} / q\right)\left(t-t_{n}\right)+\bar{c},
$$

where $\bar{c}$ is the solution of $(q+\bar{c}) f(\bar{c})=g_{T}$ and $t_{n}=b_{n}\left(q+2 c_{0}\right) / g_{T}$. If $q=0$, instead of $J_{n}$ one can use

$$
z_{n}(x, t)=2 c_{0} f\left(2 c_{0}\right)\left\{\frac{f\left(2 c_{0}\right)}{2} x^{2}-x+f\left(2 c_{0}\right) t+b_{n}\right\}+2 c_{0} .
$$

Let us give the proof in this case (for $q>0$ the proof is analogous).

Let $X_{n}=\left\{(x, t): 0 \leqslant x \leqslant b_{n}+f\left(2 c_{0}\right) t, 0 \leqslant t \leqslant T^{\prime}\right\}$. From $\left(2.20^{\prime}\right) \quad D_{n}\left(T^{\prime}\right) \subset X_{n}$, $z_{n}(x, t)$ satisfies

$$
\begin{gathered}
z_{n}(x, 0)>2 c_{0}, \quad 0 \leqslant x \leqslant b_{n}, \\
z_{n x}(0, t)=-2 c_{0} f\left(2 c_{0}\right) \leqslant c_{n x}(0, t), \quad 0 \leqslant t \leqslant T^{\prime}
\end{gathered}
$$

(this follows from the definition of $T^{\prime}$ ), and

$$
z_{n}\left(b_{n}+f\left(2 c_{0}\right) t, t\right)>2 c_{0} .
$$


Then, from the maximum principle

$$
z_{n}(x, t)>2 c_{0} \text { in } X_{n} .
$$

Now consider the difference $z_{n}-c_{n}$ and suppose that it has a negative minimum somewhere in $D_{n}\left(T^{\prime}\right)$, say in $(\bar{x}, \bar{t})$. From $(2.22),(2.16)$, and (2.23), it follows that $(\bar{x}, \bar{t})$ belongs to the free boundary, i.e., $\bar{x}=s_{n}(\bar{t})$. Moreover, it must be

$$
z_{n . x}(\bar{x}, \bar{t})-c_{n x}(\bar{x}, \bar{t})<0
$$

which implies that

$$
c_{n}(\bar{x}, \bar{t}) f\left(c_{n}(\bar{x}, \bar{t})\right)<-z_{n \cdot x}(\bar{x}, \bar{t}) .
$$

Since $z_{n x x}(x, t)>0$, it follows that

$$
c_{n}(\bar{x}, \bar{t}) f\left(c_{n}(\bar{x}, \bar{t})\right)<-z_{n x}(0, \bar{t})=2 c_{0} f\left(2 c_{0}\right)
$$

and

contradicting (2.25).

$$
z_{n}(\bar{x}, \bar{t})<c_{n}(\bar{x}, \bar{t})<2 c_{0}
$$

The requested inequality follows from the uniform bound for $z_{n}(x, t)$.

The bound for $c_{n x}$ is a consequence of the maximum principle and of the uniform boundedness of $h_{n}^{\prime}$.

Lemma 2.3. There exist an $n_{0}$ and a $T_{0}>0$ such that

$$
c_{n}(x, t) \geqslant c_{0} / 2 \text { in } D_{n}\left(T_{0}\right), n \geqslant n_{0} .
$$

Proof. We give the proof in the case $q=0$; for $q>0$ the proof is analogous. Take $n_{0}$ such that $\frac{2}{3} c_{0} f\left(\frac{2}{3} c_{0}\right) b_{n_{0}} \leqslant \frac{1}{12} c_{0}, T_{0}^{\prime}=\left(8 f\left(\frac{2}{3} c_{0}\right) f\left(2 c_{0}\right)\right)^{-1}, T_{0}^{\prime \prime}=\sup \left\{t: g(t)<-\frac{2}{3} c_{0} f\left(\frac{2}{3} c_{0}\right)\right\}$, and let $T_{0}=\inf \left\{T_{0}^{\prime}, T_{0}^{\prime \prime}\right\}$.

Comparing $c_{n}$ with the function $z(x, t)=\frac{2}{3} c_{0}\left(1-f\left(\frac{2}{3} c_{0}\right) x\right)$, using the same arguments as in Lemma 2.2, we find

$$
c_{n}(x, t)>z_{n}(x, t) \text { in } D_{n}\left(T_{0}\right), n \geqslant n_{0} .
$$

Inequality (2.26) follows from $z(x, t)>c_{0} / 2, n>n_{0}$.

Now suppose that $s_{n} \in C^{2}\left(0, T_{0}\right]$; then we have

$$
\ddot{s}_{n}(t)=f^{\prime}\left(c_{n}\left(s_{n}(t), t\right)\right)\left[c_{n_{\mathrm{v}}}\left(s_{n}(t), t\right) \dot{s}_{n}(t)+c_{n_{t}}\left(s_{n}(t), t\right)\right] .
$$

Lemma 2.4. There exists a constant $k$ depending only on $T_{0}$ (and the data $f$ and $g$ ) such that

$$
\ddot{s}_{n}(t) \leqslant k, \quad t \leqslant T_{0}
$$

for any $n \leqslant n_{0}$ ( $n_{0}$ is the same as in Lemma 2.3).

Proof. Recalling (2.27) and the estimates obtained so far, proving (2.28) reduces to finding a uniform estimate on $c_{n_{t}}$. Let $c_{n_{t}}=w+v_{n}$ where $w$ satisfies the heat equation in the quarter-plane $x>0, t>0$ with $w_{x}(0, t)=\dot{g}(t)$ and $w(x, 0)=k(x)$ for a suitable bounded $k(x)$ such that $k^{\prime}(0)=\dot{g}(0)$.

The function $v_{n}$ satisfies

$$
\begin{gathered}
v_{n_{x x}}-v_{n_{t}}=0, \quad 0<x<s_{n}(t), t>0, \\
v_{n x}(0, t)=0, \quad t>0, \\
v_{n}(x, 0)=h^{\prime \prime}(x)-k(x), \quad 0<x<b_{n},
\end{gathered}
$$


and

$$
v_{n x}+A_{n}(t) v_{n}=B_{n}(t)-W_{x}-A_{n}(t) W, \quad \text { on } x=s_{n}(t),
$$

where

$$
\begin{gathered}
A_{n}(t)=2 \dot{s}_{n}(t)+\left(q+c_{n}\left(s_{n}(t), t\right)\right) f^{\prime}\left(c_{n}\left(s_{n}(t), t\right)\right), \\
B(t)=-\left(q+c_{n}\left(s_{n}(t), t\right)\right) f^{\prime}\left(c_{n}\left(s_{n}(t), t\right)\right) c_{n \cdot x}\left(s_{n}(t), t\right) \dot{s}_{n}(t)-\dot{s}_{n}^{2}(t) c_{n x}\left(s_{n}(t), t\right) .
\end{gathered}
$$

As a consequence of the above estimates of $c_{n}, c_{n x}$, there exist two positive constants such that

$$
0<k_{1} \leqslant A_{n}, B_{n} \leqslant k_{2}
$$

for $t \leqslant T_{0}, n \geqslant n_{0}$.

Then if $\left|v_{n}\right|$ has a maximum on $x=s(t)$, its value is dominated in terms of $k_{1}, k_{2}$, and $\|w\|_{C^{1,0}\left(\bar{D}_{n}\left(T_{0}\right)\right)}$.

Moreover, $h_{n}^{\prime \prime}$ is uniformly bounded, w.r.t. $x$ and $n$, and then $\sup \left|v_{n}\right|$ in $D_{n}\left(T_{0}\right)$ is bounded by a constant independent of $n$ for $n \geqslant n_{0}$.

Let us now prove the existence of a local solution of problem $P$. First we have that the solutions $\left(c_{n}, s_{n}\right)$ of $\left(P_{n}\right)$ actually exist at least for $t \leqslant T_{0}$. We define $u_{n}(x, t)$ according to transformation (2.10) and consider the Stefan-type free boundary problem

$$
\begin{gathered}
u_{n x x}-u_{n t}=0, \quad 0<x<s_{n}(t), t>0, \\
u_{n}(x, 0)=H_{n}(x)=-\int_{x}^{b_{n}}\left[h_{n}(\eta)+q\right] d \eta, \quad 0<x<b_{n}, \\
u_{n}(0, t)=H_{n}(0)+\int_{0}^{t} g(r) d r, \quad t>0, \\
u_{n}\left(s_{n}(t), t\right)=0, \quad t>0, \\
s_{n}(0)=b_{n}, \\
\dot{s}_{n}(t)=f\left(u_{n x}\left(s_{n}(t), t\right)-q\right), \quad t>0 .
\end{gathered}
$$

This problem is of the type solved in [4]. However, it does not exactly fit the conditions for local existence and uniqueness because $f$ may not be Lipschitz continuous in 0 [for instance, $\left.f(y)=y^{\alpha}, 0<\alpha<1\right]$. If $f$ is replaced by $\phi \in C^{1}[0,+\infty)$ such that $\phi(y)=f(y)$ for $y>c_{0} / 2$, then the corresponding problem has a unique solution $\left(\tilde{u}_{n}, \tilde{s}_{n}\right)$. For this solution we can use the technique of [6] to prove that $s_{n} \in c^{2}\left(0, T_{n}\right)$ (the bootstrap argument can be used if $f \in C^{\infty}$ to get $s_{n} \in C^{\infty}$ ) and then $\tilde{c}_{n}=\tilde{u}_{n x}-q$ solves the original problem $P_{n}$ (with $\phi$ instead of $f$ ). Since our a priori estimates hold for $\left(\tilde{c}_{n}, \tilde{s}_{n}\right)$ as well, one can prove that such a solution exists at least up to $t=T_{0}$ and that the boundary conditions $\dot{s}_{n}=f\left(c_{n}\right)$ and $\dot{\tilde{s}}_{n}=\phi\left(\tilde{c}_{n}\right)$ coincide, so that $\tilde{c}_{n}, \tilde{s}_{n}$ is a solution to problem $\left(P_{n}\right)$.

Finally, let us prove the following:

Theorem 2.2. Problem $(P)$ admits a (unique) local solution. 
Proof. From the uniform estimate on $\ddot{s}_{n}$, there exists a subsequence $\left\{s_{n_{k}}\right\}$ which converges in $C^{1}\left[0, T_{0}\right]$ to some $s$. (Actually, since the data $h_{n}$ are chosen in such a way that $s_{n}<s_{m}$ if $n>m$, the whole sequence $s_{n}$ converges.) Then

$$
\begin{aligned}
\left|c_{n}(s(t), t)-c_{m}(s(t), t)\right| & \\
\leqslant & \left|c_{n}(s(t), t)-c_{n}\left(s_{n}(t), t\right)\right|+\left|c_{m}(s(t), t)-c_{m}\left(s_{m}(t), t\right)\right| \\
& +\left|c_{n}\left(s_{n}(t), t\right)-c_{m}\left(s_{m}(t), t\right)\right| \\
\leqslant & \left|G\left(T_{0}\right)\right|\left\{\left\|s-s_{n}\right\|_{C^{0}}+\left\|s-s_{m}\right\|_{C^{0}}\right\} \\
& +\left\|f^{-1}\left(\dot{s}_{n}\right)-f^{-1}\left(\dot{s}_{m}\right)\right\|_{C^{0}} \quad\left(C^{0} \equiv C^{0}\left[0, T_{0}\right]\right),
\end{aligned}
$$

with $f^{-1}$ uniformly Lipschitz continuous in the range of the functions $\dot{s}_{n}$.

Since $\left(c_{n}-c_{m}\right)_{x}(0, t)=0,\left\{c_{m}\right\}$ converges uniformly, in $D_{T_{0}}=\{(x, t): 0<x<s(t)$, $\left.0<t<T_{0}\right\}$, to a function $c(x, t)$ which solves the heat equation in $D_{T_{0}}$. Moreover,

$$
\left|\left(c_{n x}-c_{m x}\right)(s(t), t)\right| \leqslant k\left\{\left\|s-s_{n}\right\|_{C^{0}}+\left\|s-s_{m}\right\|_{C^{0}}\right\}+\left\|d^{-1}\left(\dot{s}_{n}\right)-d^{-1}\left(\dot{s}_{m}\right)\right\|
$$

with $d(y)=(q+y) f(y)$, so $\left\{c_{n x}\right\}$ converges uniformly to $c_{x}$ in $D_{T_{0}}$, and $\left(c, s, T_{0}\right)$ solves problem $(P)$.

3. Global existence and asymptotic behavior. Let us assume that $g(t)$ is negative and not decreasing, so that

$$
c_{x t}(0, t) \geqslant 0 \text {. }
$$

Consider the function $v(x, t)=c_{x}(x, t) /(c(x, t)+q)$. This function satisfies the parabolic equation

$$
v_{x x}+2 v v_{x}-v_{t}=0 \text { in } D_{T}
$$

and the boundary conditions

$$
v(s(t), t)=-\dot{s}(t) .
$$

Moreover, at each point of the free boundary such that $v_{x}(s(t), t) \neq 0$, we have

$$
-v_{t}(s(t), t) / v_{x}(s(t), t)=\dot{s}(t)+f^{\prime}(c(s(t), t))(c(s(t), t)+q)>\dot{s}(t) .
$$

As a consequence of (3.4) it follows that each of these points is an end point of a level curve $v=$ const. Repeating the arguments of [7], one finds that these curves are regular for almost every point such that (3.4) holds. Then each of these curves connects a point $(s(t), t)$ of the free boundary with a point of the fixed boundary, $(0, \bar{t})$, with $\bar{t}<t$; moreover, the $t$-coordinate is monotone all along the curve.

Now suppose that, for some $t^{\prime}$ and $t^{\prime \prime}$, we have

$$
\ddot{s}(t)>0, \quad t^{\prime}<t<t^{\prime \prime} .
$$

Since we have that $v_{x}(s(t), t)=d / d t \ln (c(s(t), t)+q)$, then inequality (3.5) implies that

$$
v_{x}(s(t), t)>0, \quad t^{\prime}<t<t^{\prime \prime},
$$

so that (3.4) is satisfied for $t^{\prime}<t<t^{\prime \prime}$. 
Let us choose a $t_{0}, t^{\prime}<t_{0}<t^{\prime \prime}$, such that the level curve $v=$ const ending at $\left(s\left(t_{0}\right), t_{0}\right)$ is a regular one, and let $x=b(t)$ be its representation and $\left(0, \bar{t}_{0}\right)$ its starting point on the fixed boundary. Let $D=\left\{(x, t): 0<x<\eta(t), \bar{t}_{0}<t<t^{\prime \prime}\right\}$, where $\eta(t)=b(t), \bar{t}_{0}<t$ $<t_{0}$, and $\eta(t)=s(t), t_{0}<t<t^{\prime \prime}$, and consider the function $v(x, t)$ restricted to $D$. We claim that $v$ cannot assume its minimum on $x=\eta(t)$; in fact, for $t<t_{0}, v(\eta(t), t)=$ $v\left(s\left(t_{0}\right), t_{0}\right)>v(s(\tau), \tau)$ for $t_{0}<\tau<t^{\prime \prime}$; moreover, for $t_{0} \leqslant t \leqslant t^{\prime \prime}$, (3.6) holds. Then there exists a time $\bar{t}$ such that the point $(0, \bar{t})$ is the minimum point of $v$ in $D$. In $(0, \bar{t})$ we have simultaneously

$$
\begin{aligned}
& v_{x}(0, \bar{t})=\left.\frac{c_{x x}(c+q)-c_{x}^{2}}{(c+q)^{2}}\right|_{(0, t)}>0, \\
& v_{t}(0, \bar{t})=\left.\frac{c_{x t}(c+q)-c_{x} c_{t}}{(c+q)^{2}}\right|_{(0, i)} \leqslant 0 .
\end{aligned}
$$

From (3.6) and $c(x, \bar{t})>0$ we have $c_{x x}(0, \bar{t})>0$. However, from (3.7) and (3.1) it follows that $c_{t}(0, \bar{t}) \leqslant 0$, contradicting the hypothesis that $\ddot{s} \geqslant 0$. We have proved

THEOREM 3.1. If $g(t) \leqslant 0$ and $\dot{g}(t) \geqslant 0$ then $\ddot{s}(t) \leqslant 0$.

Notice that the assumptions on $g(t)$ prevent $\ddot{s}$ from vanishing identically over some time interval. In fact, if the free boundary is given by $x=a t+b$, then $c$ and $c_{x}$ are given constants on it and the resulting Cauchy problem has as a unique solution $c_{x} / a(\exp (-a x$ $\left.\left.+a^{2} t+b\right)-1\right)+c$.

As a consequence of the monotonicity of $\dot{s}(t)$, one can prove the following (e.g., see [2]):

Corollary 3.2. The solution exists for any time $T>0$.

Let us now turn our attention to the asymptotic behavior of the solution.

Suppose that $\int_{0}^{+\infty} g(t) d t=-L>-\infty$; then we have

THEOREM 3.2. If $q>0$ then

$$
\lim _{t \rightarrow+\infty} s(t)=L / q .
$$

Proof. From the mass balance we have

$$
s(t)=\frac{1}{q}\left\{-\int_{0}^{s(t)} c(x, t) d x-\int_{0}^{t} g(r) d r\right\},
$$

from which it follows that

$$
s(t)<L / q \text {. }
$$

This implies that

$$
\lim _{t \rightarrow \infty} \dot{s}(t)=0
$$

and consequently

$$
\lim _{t \rightarrow \infty} c(x, t)=0, \quad \text { uniformly w.r.t. } x,
$$

and (3.8) follows from (3.9). 
THEOREM 3.3. If $q=0$ then

$$
\lim _{t \rightarrow \infty} s(t)=+\infty, \quad \lim _{t \rightarrow \infty} \dot{s}(t)=0 .
$$

Proof. From the mass balance we have

$$
\int_{0}^{s(t)} c(x, t) d x=-\int_{0}^{t} g(r) d r .
$$

Since $\ddot{s}(t) \leqslant 0$, there exists $\lim _{t \rightarrow \infty} \dot{s}(t)$ and then $\lim _{t \rightarrow \infty} c(s(t), t)$. Suppose that $\lim _{t \rightarrow \infty} s(t)<+\infty$, then $\lim _{t \rightarrow \infty} c(x, t)=0$ uniformly w.r.t. $x$ and from (3.11) we obtain a contradiction.

Moreover, we have

$$
-\int_{0}^{t} g(r) d r \geqslant f^{-1}(\dot{s}(t)) s(t)
$$

from which the second limit of (3.10) follows immediately.

The way $s(t)$ grows to infinity can be estimated if we assume $f(z)=\beta z^{m}, \beta>0$, $m>0$.

THEOREM 3.4. If $q=0$ and $f(z)=\beta z^{m}, \beta>0, m>0$, then

$$
s(t) \leqslant\left\{(m+1) \beta \int_{0}^{t}\left(\int_{0}^{r} g(\eta) d \eta\right)^{m} d r\right\}^{1 / m+1}
$$

if $\int_{0}^{+\infty} g(t) d t=-L$ then $s(t) \leqslant$ const. $t^{1 / m+1}$.

Proof. Inequality (3.12) follows immediately by integrating

$$
-\int_{0}^{t} g(r) d r \geqslant \beta^{-1 / m} \dot{S}(t)^{1 / m} s(t) .
$$

\section{REFERENCES}

[1] G. Astarita and G. C. Sarti, A class of mathematical models for sorption of swelling solvents into glassy polymers, Polymer Eng. Sci. 18, 388-395 (1978)

[2] E. Comparini and R. Ricci. On the swelling of a glassy polymer in contact with a well-stirred solvent, Math. Methods Appl. Sci. 7, 238-250 (1985)

[3] A. Fasano, G. H. Mayer, and M. Primicerio, On a problem in polymer industry: Theoretical and numerical investigation, SIAM J. Math. Anal. 17, 945-960 (1986)

[4] A. Fasano and M. Primicerio, Free boundary problems for nonlinear parabolic equations with nonlinear free boundary conditions, J. Math. Anal. Appl. 72, 247-273 (1979)

[5] G. C. Sarti, Solvent osmotic stresses and the prediction of case II transport kinetics, Polymer 20, 825-832 (1979)

[6] D. G. Schaeffer, A new proof of the infinite differentiability of the free boundary in the Stefan problem. J. Differential Equations 20, 266-269 (1976)

[7] A. Friedman and R. Jensen, Convexity of the free boundary in the Stefan problem and in the dam problem, Arch. Rat. Mech. Anal. 67, 1-24 (1977) 